Contemporary Mathematics

Volume 00, 1997

\title{
Type A Fusion Rules from Elementary Group Theory
}

\author{
Alex J. Feingold, Michael D. Weiner
}

\section{Introduction}

Fusion rules play a very important role in conformal field theory $[\mathbf{F u}]$, in the representation theory of vertex operator algebras $[\mathbf{F H L}]$, and in quite a few other areas. We are interested in understanding fusion rules from a combinatorial point of view, using very elementary aspects of group theory. Several authors have also investigated fusion rules from a combinatorial point of view [BWM,BKMW,T], but we believe our approach is unique. We have used this approach successfully in $[\mathbf{A F W}]$ to obtain all of the $(p, q)$-minimal model fusion rules ([Wa]) from elementary 2-groups. The $(p, q)$-minimal models are a certain series of highest weight representations of the Virasoro algebra $([\mathbf{K R}])$ which also have the structure of a vertex operator algebra $([\mathbf{F L M}])$, and modules for it $([\mathbf{F Z}])$. The Goddard-KentOlive coset construction $[\mathbf{G O}]$ can be used to get the unitary $(p, p+1)$-minimal models from representations of the affine Kac-Moody Lie algebra $\hat{\mathbf{g}}$ where $\mathbf{g}=s l_{2}$ is the finite dimensional Lie algebra of type $A_{1}$. Knowing that, we were not surprised to notice that the approach in [AFW] would give the fusion rules for level $k$ representations of the affine $A_{1}$ algebra from elementary 2 -groups. It is perhaps a bit more surprising that the technique worked for all of the $(p, q)$-minimal models, not just the unitary ones coming from affine $A_{1}$ coset constructions. Until now our approach has only given fusion rules which have the special property that the fusion coefficients $N_{i j}^{k}$ are in $\{0,1\}$. In order for our approach to be of greater significance, we had to find a way to incorporate the higher multiplicities which can occur even in the case of affine $A_{2}$. In this paper we achieve that goal in such a way as to see how the simpler special cases only have multiplicity one or zero. We cannot yet give the complete answer for all affine type A algebras, but we present here for all levels, our approach for ranks $N=1$ and $N=2$.

The inspiration for this work on fusion rules comes from certain explicit constructions of vertex operator algebras (VOAs), their modules, and spaces of intertwining operators $([\mathbf{F e}, \mathbf{F F R}, \mathbf{F R W}, \mathbf{W e}])$ which can all be unified into one large algebraic system. When the VOA and its modules are indexed by a finite abelian group, $G$, and certain technical conditions are satisfied, we call such a system a

1991 Mathematics Subject Classification. Primary 17B67, 17B65, 81T40; Secondary 81R10, $05 \mathrm{E} 10$.

We wish to thank Matthias Beck, Jürgen Fuchs and Thomas Zaslavsky for valuable advice. 
vertex operator para-algebra (VOPA). A somewhat more general structure, called an abelian intertwining algebra, was defined in $[\mathbf{D L}]$. In those systems the fusion rules are given precisely by the group algebra of $G$. This is the situation for level one modules of an untwisted affine Kac-Moody Lie algebra $\hat{\mathrm{g}}$, where the group $G$ is the quotient of the weight lattice by the root lattice of the underlying finite dimensional Lie algebra, g. For example, in $[\mathbf{F F R}]$ the spinor construction of the four level one modules for the orthogonal affine Kac-Moody Lie algebra of type $D_{4}^{(1)}$ was used to construct a vertex operator para-algebra with $G=\mathbf{Z}_{2} \times \mathbf{Z}_{2}$. A similar phenomenon occurred in $[\mathbf{W e}]$, where a vertex operator para-algebra was constructed from the four level $-\frac{1}{2}$ modules of the symplectic affine Kac-Moody Lie algebras of type $C_{n}^{(1)}$, but the group $G$ could be taken as either $\mathbf{Z}_{4}$ or $\mathbf{Z}_{2} \times \mathbf{Z}_{2}$. We were searching for the appropriate generalization of this idea which would include the VOAs and their modules coming from higher level representations of Kac-Moody Lie algebras $([\mathbf{F Z}, \mathbf{T K}])$ and the $(p, q)$-minimal models. A key aspect of the generalization would have to incorporate the fusion rules which can come from spaces of intertwining operators which are of dimension greater than one. We first looked at the spinor construction of the $(3,4)$-minimal model in $[\mathbf{F R W}]$, that is, the representations of the Virasoro algebra with central element $c=\frac{1}{2}$ and with $h=0, h=\frac{1}{2}$ and $h=\frac{1}{16}$, which naturally has two copies of the $h=\frac{1}{16}$ module. Let us denote those modules by $[0],\left[\frac{1}{2}\right],\left[\frac{1}{16}\right]_{1}$ and $\left[\frac{1}{16}\right]_{2}$, respectively. Having two copies of $\left[\frac{1}{16}\right]$ allows the usual Ising model fusion rules to be replaced by the group $\mathbf{Z}_{4}$ or by $\mathbf{Z}_{2} \times \mathbf{Z}_{2}$ because there are intertwining operators labelled by independent vectors which act on the two $\left[\frac{1}{16}\right]$ modules, sending them to either $[0]$ or $\left[\frac{1}{2}\right]$. This is different from the usual situation, where one intertwining operator labelled by one vector from the one $h=\frac{1}{16}$ module stands for a two-dimensional space of operators sending that $\left[\frac{1}{16}\right]$ module to a linear combination of $[0]$ and $\left[\frac{1}{2}\right]$. This paper and $[\mathbf{A F W}]$ are the result of looking for other situations where having more copies of each irreducible VOA module, each copy labelled by an element of a finite abelian group, allows the fusion rules to be "pulled apart", showing an underlying group algebra.

One suspects that such an explanation of fusion rules for the higher level KacMoody algebras should come from the level one para-algebra structure (or perhaps, abelian intertwining algebra structure). For example, in type $A_{N-1}$, where the weight lattice modulo the root lattice is $\mathbf{Z}_{N}$, the direct sum of the $N$ level one fundamental modules should form an abelian intertwining algebra, $\mathcal{V}$. The $k^{\text {th }}$ tensor power of $\mathcal{V}$ contains all level $k$ modules, and is naturally an abelian intertwining algebra graded by the group $\mathbf{Z}_{N}^{k}$. Then the fusion rules among those level $k$ modules must be strongly influenced by that group $\mathbf{Z}_{N}^{k}$, but a precise understanding of how is still lacking. The fact that the symmetric group $S_{k}$ acts naturally on the $k^{\text {th }}$ tensor power of $\mathcal{V}$ is certainly relevant, and plays a crucial role in our current paper.

A brief summary of our results is as follows. Let $\hat{g}$ be the affine algebra of type $A_{N-1}^{(1)}$ built from $\mathbf{g}=s l_{N}$. Let $\lambda_{1}, \cdots, \lambda_{N-1}$ denote the fundamental weights of $\mathbf{g}$, and let $P^{+}$denote the dominant integral weights of $\mathbf{g}$. The irreducible modules $\hat{V}_{\Lambda}$ for $\hat{g}$ of level $k \geq 1$ are indexed by a dominant integral highest weight $\Lambda=k c+\lambda$ 
where

$$
\lambda=\sum_{j=1}^{N-1} a_{j} \lambda_{j} \in P^{+}
$$

satisfies the "level $k$ condition" $\sum_{j=1}^{N-1} a_{j} \leq k$. Then the irreducible modules on level $k$ are in one-to-one correspondence with the set of $N$-tuples of nonnegative integers $\left(i_{0}, i_{1}, \cdots, i_{N-1}\right)$ whose sum is $k$. Such an $N$-tuple corresponds to

$$
\Lambda=k c+i_{1} \lambda_{1}+\cdots+i_{N-1} \lambda_{N-1} .
$$

Fix level $k \geq 1$ and write the product in the fusion algebra for such modules as

$$
[\lambda] \times[\mu]=\sum_{\nu \in P^{+}} N_{[\lambda],[\mu]}^{[\nu]}[\nu] .
$$

The distinguished module, [0], is an identity element for this product, and for each $[\lambda]$ there is a distinguished conjugate $\left[\lambda^{+}\right]$such that $N_{[\lambda],[\mu]}^{[0]}=\delta_{\mu, \lambda^{+}}$. A knowledge of the fusion coefficients, $N_{[\lambda],[\mu]}^{[\nu]}$, is equivalent to a knowledge of the completely symmetric coefficients

$$
N_{[\lambda],[\mu],[\nu]}=N_{[\lambda],[\mu]}^{\left[\nu^{+}\right]} .
$$

Let $G=\mathbf{Z}_{N}^{k}$ and let the symmetric group $S_{k}$ act on $G$ by permuting the $k$ tuples. For $a \in G$, let $[a]$ denote the orbit of $a$ under this action and let $\mathcal{O}$ be the set of all such orbits. These orbits are precisely the subsets

$$
P\left(i_{0}, i_{1}, \ldots, i_{N-1}\right)=\left\{x \in \mathbf{Z}_{N}^{k} \mid j \text { occurs exactly } i_{j} \text { times in } x, 0 \leq j \leq N-1\right\}
$$

where $\left(i_{0}, i_{1}, \cdots, i_{N-1}\right)$ is any $N$-tuple of nonnegative integers such that $i_{0}+i_{1}+$ $\cdots+i_{N-1}=k$. We now have a bijection between $\mathcal{O}$ and the set of level $k$ modules. For $[a],[b],[c] \in \mathcal{O}$ we believe the fusion coefficients $N_{[a],[b]}^{[c]}$ have a combinatorial description in terms of the group $G$. The conjugate of $[c]$ is $[-c]$ and we prefer to study the symmetric coefficients

$$
N_{[a],[b],[c]}=N_{[a],[b]}^{[-c]} .
$$

The combinatorial question which we were led to investigate is as follows. For any $[a],[b],[c] \in \mathcal{O}$, the group $S_{k}$ acts on

$$
T([a],[b],[c])=\{(x, y, z) \in[a] \times[b] \times[c] \mid x+y+z=0\}
$$

which decomposes into a finite number of orbits under that action. Let the number of such orbits be denoted by $M([a],[b],[c])$. For ranks $N=2$ and $N=3$ we determine $M([a],[b],[c])$ and show how it is related to $N_{[a],[b],[c]}$. In future work we will try to give the relationship for all ranks. We have the following results.

THEOREM. For $N=2$, for any integral level $k \geq 1$, with notation as above, we have

$$
M([a],[b],[c])=N_{[a],[b],[c]} \cdot
$$

THEOREM. For $N=3$, for any integral level $k \geq 1$, with notation as above, we have

$$
M([a],[b],[c])=\left(\begin{array}{c}
N_{[a],[b],[c]}+1 \\
2
\end{array}\right) .
$$




\section{Background}

We use the definition of fusion algebra given in $[\mathbf{F u}]$. A fusion algebra $F$ is a finite dimensional commutative associative algebra over $\mathbf{Q}$ with some basis $B=\left\{x_{a} \mid a \in A\right\}$ such that the structure constants $N_{a, b}^{c}$ defined by

$$
x_{a} \cdot x_{b}=\sum_{c \in A} N_{a, b}^{c} x_{c}
$$

are non-negative integers. It is also required that there be a distinguished index $\Omega \in A$ with the following properties. Define a matrix $C=\left[C_{a, b}\right]$ by $C_{a, b}=N_{a, b}^{\Omega}$ and define an associated "conjugation" map $\mathcal{C}: F \rightarrow F$ by

$$
\mathcal{C}\left(x_{a}\right)=\sum_{b \in A} C_{a, b} x_{b}
$$

It is required that $\mathcal{C}$ be an involutive automorphism of $F$. This implies that $\mathcal{C}^{2}=I_{F}$ is the identity on $F$, so $C^{2}=I$ is the identity matrix. Because $0 \leq N_{a, b}^{c} \in \mathbf{Z}$, we get that either $C=I$ or $C$ must be an order 2 permutation matrix, that is, there is a permutation $\sigma: A \rightarrow A$ with $\sigma^{2}=1$ and

$$
C_{a, b}=\delta_{a, \sigma(b)} .
$$

Since $\mathcal{C}$ is an automorphism, we must also have $\mathcal{C}\left(x_{a}\right) \cdot \mathcal{C}\left(x_{b}\right)=\mathcal{C}\left(x_{a} \cdot x_{b}\right)$, that is,

$$
x_{\sigma(a)} \cdot x_{\sigma(b)}=\sum_{c \in A} N_{a, b}^{c} x_{\sigma(c)}
$$

which means that $N_{\sigma(a), \sigma(b)}^{\sigma(c)}=N_{a, b}^{c}$. Sometimes we may write $\sigma(a)=a^{+}$and call $x_{a^{+}}$the conjugate of $x_{a}$. One may use it to define the non-negative integers

$$
N_{a, b, c}=N_{a, b}^{c^{+}}
$$

which, by commutativity and associativity of the structure constants, are completely symmetric in $a, b$ and $c$. Furthermore, one finds that

$$
\begin{aligned}
N_{\Omega, b}^{c} & =N_{\Omega, b, c^{+}}=N_{b, c^{+}, \Omega} \\
& =N_{b, c^{+}}^{\Omega^{+}}=N_{b^{+}, c}^{\Omega} \\
& =C_{b^{+}, c}=\delta_{b, c}
\end{aligned}
$$

which means that $x_{\Omega}$ is an identity element for multiplication in $F$, usually written $x_{\Omega}=1$. It also follows that $\Omega^{+}=\Omega$.

In $[\mathbf{A F W}]$ we introduced the idea of covering a fusion algebra by a finite abelian group and proved that the $(p, q)$-minimal model fusion algebra, which comes from the discrete series of $0<c<1$ representations of the Virasoro algebra, can be covered by the group $Z_{2}^{p+q-5}$. The basic idea, which is only set up to handle fusion algebras whose fusion coefficients $N_{i j}^{k}$ are in $\{0,1\}$, is as follows.

Definition. Let $(G,+, 0)$ be a finite abelian group and let $G=P_{0} \cup P_{1} \cup \ldots \cup P_{N}$ be a partition into $N$ disjoint subsets with $P_{0}=\{0\}$. Let $W$ be an $N$-dimensional 
vector space over $\mathbf{Q}$ with basis $P=\left\{P_{0}, P_{1}, \ldots, P_{N}\right\}$ and define a bilinear multiplication on $W$ by the formula

$$
P_{i} * P_{j}=\sum_{k \in T(i, j)} P_{k}
$$

where $T(i, j)=\left\{k \mid \exists a \in P_{i}, \exists b \in P_{j}, a+b \in P_{k}\right\}$. We say that such a partition is associative if the product $*$ is associative. We say that a group $G$ covers a fusion algebra if there is an associative partition $P$ of $G$ and a bijection $\Phi$ between $A$ and $P$ which gives an algebra isomorphism between $F$ and $W$ such that $\Phi(\Omega)=P_{0}$.

There are certainly examples of groups with partitions which are not associative. Perhaps it is surprising that there are any interesting examples of groups with associative partitions. As an example of a nonassociative partition, let $G=\mathbf{Z}_{5}$, and let $P_{0}=\{0\}, P_{1}=\{1,2\}$ and $P_{2}=\{3,4\}$. The multiplication table for the corresponding $W$ is as follows.

Table 1: A nonassociative partition for $\mathbf{Z}_{5}$.

\begin{tabular}{|c|c|c|c|}
\hline$P_{i} * P_{j}$ & $P_{0}$ & $P_{1}$ & $P_{2}$ \\
\hline$P_{0}$ & $P_{0}$ & $P_{1}$ & $P_{2}$ \\
\hline$P_{1}$ & $P_{1}$ & $P_{1}+P_{2}$ & $P_{0}+P_{1}+P_{2}$ \\
\hline$P_{2}$ & $P_{2}$ & $P_{0}+P_{1}+P_{2}$ & $P_{1}+P_{2}$ \\
\hline
\end{tabular}

We then compute

$\left(P_{1} * P_{2}\right) * P_{2}=\left(P_{0}+P_{1}+P_{2}\right) * P_{2}=P_{2}+\left(P_{0}+P_{1}+P_{2}\right)+\left(P_{1}+P_{2}\right)=P_{0}+2 P_{1}+3 P_{2}$

and

$$
P_{1} *\left(P_{2} * P_{2}\right)=P_{1} *\left(P_{1}+P_{2}\right)=\left(P_{1}+P_{2}\right)+\left(P_{0}+P_{1}+P_{2}\right)=P_{0}+2 P_{1}+2 P_{2}
$$

so $\left(P_{1} * P_{2}\right) * P_{2} \neq P_{1} *\left(P_{2} * P_{2}\right)$.

The fusion rule tables presented here were produced by the computer program of Bert Schellekens, called "Kac", available from his webpage [http://norma.nikhet.nI/ $\sim t 58 /$. As an example of a nontrivial fusion algebra which can be covered by a group, let the $\mathcal{W}$ algebra coming from the coset construction of

$$
\frac{S U(N)_{r} \otimes S U(N)_{s}}{S U(N)_{r+s}}
$$

be denoted by $\mathcal{W}_{N}(r, s)$. The fusion rules for $\mathcal{W}_{3}(1,1)$ are as follows. 
Table 2: Fusion rules for $\mathcal{W}_{3}(1,1)$.

\begin{tabular}{|c|c|c|c|c|c|c|}
\hline$[\mathrm{a}] \times[\mathrm{b}]$ & {$[0]$} & {$[1]$} & {$[2]$} & {$[3]$} & {$[4]$} & {$[5]$} \\
\hline$[0]$ & {$[0]$} & {$[1]$} & {$[2]$} & {$[3]$} & {$[4]$} & {$[5]$} \\
\hline$[1]$ & & {$[0]+[1]$} & {$[3]$} & {$[2]+[3]$} & {$[5]$} & {$[4]+[5]$} \\
\hline$[2]$ & & & {$[4]$} & {$[5]$} & {$[0]$} & {$[1]$} \\
\hline$[3]$ & & & & {$[4]+[5]$} & {$[1]$} & {$[0]+[1]$} \\
\hline$[4]$ & & & & & {$[2]$} & {$[3]$} \\
\hline$[5]$ & & & & & & {$[2]+[3]$} \\
\hline
\end{tabular}

Note that $\{[0],[2],[4]\}$ forms a subgroup isomorphic to $\mathbf{Z}_{3}$. We find that $\mathbf{Z}_{3}^{2}$ covers these fusion rules as follows:

$$
\begin{array}{rlrl}
\{(0,0)\} & \leftrightarrow[0] & \{(1,2),(2,1)\} \leftrightarrow[1] \quad\{(1,1)\} \leftrightarrow[2] \\
\{(0,2),(2,0)\} & \leftrightarrow[3] \quad\{(2,2)\} \leftrightarrow[4] \quad\{(1,0),(0,1)\} \leftrightarrow[5]
\end{array}
$$

A multiplication table which illustrates exactly how $\mathbf{Z}_{3}^{2}$ covers the $W_{3}(1,1)$ fusion rules is as follows.

\begin{tabular}{|c|c|c|c|c|c|c|}
\hline$[\mathrm{a}] \times[\mathrm{b}]$ & $(0,0)$ & $(1,2),(2,1)$ & $(1,1)$ & $(0,2),(2,0)$ & $\overline{(2,2)}$ & $(1,0),(0,1)$ \\
\hline$(0,0)$ & $\overline{(0,0)}$ & $(1,2),(2,1)$ & $(1,1)$ & $(0,2),(2,0)$ & $\overline{(2,2)}$ & $(1,0),(0,1)$ \\
\hline $\begin{array}{l}(1,2) \\
(2,1)\end{array}$ & & $\begin{array}{l}(2,1),(0,0) \\
(0,0),(1,2)\end{array}$ & $\begin{array}{l}(2,0) \\
(0,2)\end{array}$ & $\begin{array}{l}(1,1),(2,0) \\
(0,2),(1,1)\end{array}$ & $\begin{array}{l}(0,1) \\
(1,0)\end{array}$ & $\begin{array}{l}(2,2),(0,1) \\
(1,0),(2,2)\end{array}$ \\
\hline$(1,1)$ & & & $(2,2)$ & $(1,0),(0,1)$ & $(0,0)$ & $(2,1),(1,2)$ \\
\hline $\begin{array}{l}(0,2) \\
(2,0)\end{array}$ & & & & $\begin{array}{l}(0,1),(2,2) \\
(2,2),(1,0)\end{array}$ & $\begin{array}{l}(2,1) \\
(1,2)\end{array}$ & $\begin{array}{l}(1,2),(0,0) \\
(0,0),(2,1)\end{array}$ \\
\hline$(2,2)$ & & & & & $(1,1)$ & $(0,2),(2,0)$ \\
\hline $\begin{array}{l}(1,0) \\
(0,1)\end{array}$ & & & & & & $\begin{array}{l}(2,0),(1,1) \\
(1,1),(0,2)\end{array}$ \\
\hline
\end{tabular}

Table 3: $\mathbf{Z}_{3}^{2}$ covering the $\mathcal{W}_{3}(1,1)$ fusion rules.

The simplest series of fusion algebras comes from the affine algebra $\hat{g}$ of type $A_{1}^{(1)}$ built on $\mathbf{g}=s l_{2}$. There are $k+1$ modules $\hat{V}_{a}$ for $\hat{g}$ of level $k \geq 1$ which can be indexed by "spin" $a \in \frac{1}{2} \mathbf{Z}$ with $0 \leq a \leq \frac{k}{2}$. The conformal weight of the highest weight vector in $\hat{V}_{a}$ is

$$
\Delta_{a}=\frac{a(a+1)}{k+2}
$$

The g-submodule $V_{a}$ of $\hat{V}_{a}$ generated by a highest weight vector is of dimension $2 a+1$. There is a simple formula for the decomposition of the tensor product $V_{a} \otimes V_{b}$ into the direct sum of $\mathbf{g}$-modules $V_{c}$,

$$
V_{a} \otimes V_{b}=\sum_{|a-b| \leq c \leq a+b} V_{c}
$$


where the sum is only taken over those $c \in \frac{1}{2} \mathbf{Z}$ such that $a+b+c \in \mathbf{Z}$. This decomposition is independent of the level $k$ and is part of the basic representation theory of $s l_{2}$. It is remarkable that the fusion rules for level $k$ are obtained simply by truncating the above summation. The following formula for the $A_{1}^{(1)}$ level $k$ fusion rules is well-known $([\mathbf{T K}])$ :

$$
N_{a, b}^{c}=1 \quad \text { if } \quad|a-b| \leq c \leq a+b, \quad a+b+c \in \mathbf{Z}, \quad a+b+c \leq k,
$$

and $N_{a, b}^{c}=0$ otherwise. The conditions above imply that $c \leq \frac{k}{2}$.

We can also express these fusion rules in the following simple way. Re-index the $k+1$ modules $\hat{V}_{a}$ on level $k$ by $m=2 a+1 \in \mathbf{Z}$ with $1 \leq m \leq k+1$. Then $m=\operatorname{dim}\left(V_{a}\right)$ and we write $\hat{V}_{a}=\hat{V}(2 a+1)=\hat{V}(m)$. Let $p=\bar{k}+2$.

DEFINITION. For integer $p \geq 2$, we say that the triple of integers $\left(m, m^{\prime}, m^{\prime \prime}\right)$ is p-admissible when $0<m, m^{\prime}, m^{\prime \prime}<p$, the sum $m+m^{\prime}+m^{\prime \prime}<2 p$ is odd, and the "triangle" inequalities $m<m^{\prime}+m^{\prime \prime}, m^{\prime}<m+m^{\prime \prime}$, and $m^{\prime \prime}<m+m^{\prime}$ are satisfied.

Then the fusion rules are given by $N_{m, m^{\prime}}^{m^{\prime \prime}}=1$ if $\left(m, m^{\prime}, m^{\prime \prime}\right)$ is p-admissible, $N_{m, m^{\prime}}^{m^{\prime \prime}}=0$ otherwise.

The proof in $[\mathbf{A F W}]$ is easily modified to show that the $A_{1}^{(1)}$ fusion ring for level $k$ is covered by the group $\mathbf{Z}_{2}^{k}$.

THEOREM 1. The level $k$ fusion rules for $\hat{g}$ of type $A_{1}^{(1)}$ define a fusion algebra $F$ with $A=\{m \in \mathbf{Z} \mid 1 \leq m \leq k+1\}$ with distinguished element $\Omega$ being $m=1$ and the conjugate of $m$ is $m$. The the fusion algebra $F$ is covered by the elementary abelian 2-group $G=\mathbf{Z}_{2}^{k}$ with partition given by

$$
P_{i}=\{g \in G \mid \text { exactly } i \text { coordinates of } g \text { are } 1\} \text { for } 0 \leq i \leq k .
$$

We illustrate how this theorem works in the following tables. We have used a labelling scheme where on level $k$ the $k+1$ generators of the fusion ring are denoted by $[i]$ for $0 \leq i \leq k$ and the distinguished element $\Omega$ is [0]. Following each fusion rule table is a table showing how the group $\mathbf{Z}_{2}^{k}$ covers it.

Table 4: Fusion Table for $A_{1}$ of level $k=2$.

\begin{tabular}{|c|c|c|c|}
\hline$[\mathrm{i}] \times[\mathrm{j}]$ & {$[0]$} & {$[1]$} & {$[2]$} \\
\hline$[0]$ & {$[0]$} & {$[1]$} & {$[2]$} \\
\hline$[1]$ & & {$[0]$} & {$[2]$} \\
\hline$[2]$ & & & {$[0]+[1]$} \\
\hline
\end{tabular}

Table 5: Group $\mathbf{Z}_{2}^{2}$ covering the Fusion Table for $A_{1}$ of level $k=2$.

\begin{tabular}{|c|c|c|c|}
\hline$[\mathrm{i}] \times[\mathrm{j}]$ & $(0,0)$ & $(1,1)$ & $(1,0),(0,1)$ \\
\hline$(0,0)$ & $(0,0)$ & $(1,1)$ & $(1,0),(0,1)$ \\
\hline$(1,1)$ & & $(0,0)$ & $(0,1),(1,0)$ \\
\hline$(1,0),(0,1)$ & & & $\begin{array}{l}(0,0),(1,1) \\
(1,1),(0,0)\end{array}$ \\
\hline
\end{tabular}


Table 6: Fusion Table for $A_{1}$ of level $k=3$.

\begin{tabular}{|c|c|c|c|c|}
\hline$[\mathrm{i}] \times[\mathrm{j}]$ & {$[0]$} & {$[1]$} & {$[2]$} & {$[3]$} \\
\hline$[0]$ & {$[0]$} & {$[1]$} & {$[2]$} & {$[3]$} \\
\hline$[1]$ & & {$[0]$} & {$[3]$} & {$[2]$} \\
\hline$[2]$ & & & {$[0]+[2]$} & {$[1]+[3]$} \\
\hline$[3]$ & & & & {$[0]+[2]$} \\
\hline
\end{tabular}

Table 7: Group $\mathbf{Z}_{2}^{3}$ covering the Fusion Table for $A_{1}$ of level $k=3$.

\begin{tabular}{|c|c|c|c|c|}
\hline$[\mathrm{i}] \times[\mathrm{j}]$ & $(0,0,0)$ & $(1,1,1)$ & $(0,1,1),(1,0,1),(1,1,0)$ & $(1,0,0),(0,1,0),(0,0,1)$ \\
\hline$(0,0,0)$ & $(0,0,0)$ & $(1,1,1)$ & $(1,0,0),(0,1,0),(0,0,1)$ & $(0,1,1),(1,0,1),(1,1,0)$ \\
\hline$(1,1,1)$ & & $(0,0,0)$ & $(0,1,1),(1,0,1),(1,1,0)$ & $(1,0,0),(0,1,0),(0,0,1)$ \\
\hline$(0,1,1)$ & & & $(0,0,0),(1,1,0),(1,0,1)$ & $(1,1,1),(0,0,1),(0,1,0)$ \\
$(1,0,1)$ & & & $(1,1,0),(0,0,0),(0,1,1)$ & $(0,0,1),(1,1,1),(1,0,0)$ \\
$(1,1,0)$ & & & $(1,0,1),(0,1,1),(0,0,0)$ & $(1,0,0),(1,0,0),(1,1,1)$ \\
\hline $\begin{array}{c}1,0,0) \\
0,1,0) \\
(0,0,1)\end{array}$ & & & & \\
\hline
\end{tabular}

The next simplest series of fusion algebras comes from the affine algebra $\hat{g}$ of type $A_{2}^{(1)}$ built from $\mathbf{g}=s l_{3}$. There are $(k+1)(k+2) / 2$ irreducible modules $\hat{V}_{\Lambda}$ for $\hat{g}$ of level $k \geq 1$ which can be indexed by a dominant integral highest weight $\Lambda=k c+\lambda$ where $\lambda=a_{1} \lambda_{1}+a_{2} \lambda_{2} \in P^{+}$is a dominant integral weight for the finite dimensional algebra g satisfying the "level $k$ condition" $a_{1}+a_{2} \leq k$ for $0 \leq a_{i} \in \mathbf{Z}$. The irreducible $\mathrm{g}$-submodule $V_{\lambda}$ of $\hat{V}_{\Lambda}$ generated by a highest weight vector is of dimension $\left(a_{1}+1\right)\left(a_{2}+1\right)\left(a_{1}+a_{2}+2\right) / 2$. The decomposition of the tensor product of irreducible $\mathbf{g}$-modules

$$
V_{\lambda} \otimes V_{\mu}=\sum_{\nu \in P^{+}} \operatorname{Mult}_{\lambda, \mu}^{\nu} V_{\nu}
$$

into the direct sum of irreducible $\mathbf{g}$-modules includes multiplicities. This decomposition is independent of the level $k$ and is part of the basic representation theory of $s l_{3}$. The fusion rules for level $k$ are obtained by a rather subtle truncation of the above summation. The tensor product multiplicity, $M u l t_{\lambda, \mu}^{\nu}$, is equal to the multiplicity of the trivial module $V_{0}$ in the triple tensor product $V_{\lambda} \otimes V_{\mu} \otimes V_{\nu^{+}}$, where $V_{\nu^{+}}$is the contragredient module of $V_{\nu}$. This means that $\nu^{+}$is the highest weight of the dual space of $V_{\nu}$, so $\nu^{+}$is the negative of the lowest weight of $V_{\nu}$. Then

$$
\operatorname{Mult}_{\lambda, \mu, \nu}=\operatorname{Mult}_{\lambda, \mu}^{\nu^{+}}
$$

is the multiplicity of $V_{0}$ in the triple tensor product $V_{\lambda} \otimes V_{\mu} \otimes V_{\nu}$. This number is clearly symmetric in $\lambda, \mu$ and $\nu$.

We have the following result from [BMW] giving the $A_{2}$ tensor product multiplicities and the fusion rules on level $k$. 
Theorem 2. [BMW] Let $\lambda=a_{1} \lambda_{1}+a_{2} \lambda_{2}, \mu=b_{1} \lambda_{1}+b_{2} \lambda_{2}$, and $\nu=c_{1} \lambda_{1}+$ $c_{2} \lambda_{2}$ be dominant integral weights for $\mathbf{g}=s l_{3}$, and let

$\mathcal{A}=\frac{1}{3}\left(2\left(a_{1}+b_{1}+c_{1}\right)+a_{2}+b_{2}+c_{2}\right), \quad \mathcal{B}=\frac{1}{3}\left(a_{1}+b_{1}+c_{1}+2\left(a_{2}+b_{2}+c_{2}\right)\right)$.

Define $k_{0}^{\max }=\min (\mathcal{A}, \mathcal{B})$ and

$$
k_{0}^{\text {min }}=\max \left(a_{1}+a_{2}, b_{1}+b_{2}, c_{1}+c_{2}, \mathcal{A}-\min \left(a_{1}, b_{1}, c_{1}\right), \mathcal{B}-\min \left(a_{2}, b_{2}, c_{2}\right)\right) .
$$

Let $\delta=1$ if $k_{0}^{\max } \geq k_{0}^{\min }$ and $\mathcal{A}, \mathcal{B} \in \mathbf{Z}$, and let $\delta=0$ otherwise. Denote the consecutive integers $k_{0}^{\min }, k_{0}^{\min }+1, \ldots, k_{0}^{\max }$ by $k_{0}^{(i)}$, for $1 \leq i \leq M$. Then we have the tensor product multiplicity

$$
M=\text { Mult }_{\lambda, \mu, \nu}=\left(k_{0}^{\max }-k_{0}^{\min }+1\right) \delta
$$

and the fusion rule coefficient on level $k$ is

$$
N_{\lambda, \mu, \nu}^{(k)}= \begin{cases}\max (i) & \text { such that } k \geq k_{0}^{(i)} \text { and } M u l t_{\lambda, \mu, \nu} \neq 0, \\ 0 & \text { if } k<k_{0}^{(1)} \text { or Mult } \text { or, }_{\lambda, \nu}=0\end{cases}
$$

so if $M u l t_{\lambda, \mu, \nu} \neq 0$ and $k_{0}^{\min } \leq k$ then $N_{\lambda, \mu, \nu}^{(k)}=\min \left(k_{0}^{\max }, k\right)-k_{0}^{\min }+1$.

The fusion algebra for $A_{2}^{(1)}$ on level $k=1$ is rather trivial, with three modules whose fusion rules are given exactly by the group $\mathbf{Z}_{3}$, which is the weight lattice modulo the root lattice of type $A_{2}$. The fusion algebra for $A_{2}^{(1)}$ on level $k=2$, and its covering by the group $\mathbf{Z}_{3}^{2}$, are in the next two tables.

Table 8: Fusion Table for $A_{2}$ of level $k=2$.

\begin{tabular}{|c|c|c|c|c|c|c|}
\hline$[\mathrm{i}] \times[\mathrm{j}]$ & {$[0]$} & {$[1]$} & {$[2]$} & {$[3]$} & {$[4]$} & {$[5]$} \\
\hline$[0]$ & {$[0]$} & {$[1]$} & {$[2]$} & {$[3]$} & {$[4]$} & {$[5]$} \\
\hline$[1]$ & & {$[2]$} & {$[0]$} & {$[4]$} & {$[5]$} & {$[3]$} \\
\hline$[2]$ & & & {$[1]$} & {$[5]$} & {$[3]$} & {$[4]$} \\
\hline$[3]$ & & & & {$[0]+[3]$} & {$[1]+[4]$} & {$[2]+[5]$} \\
\hline$[4]$ & & & & & {$[2]+[5]$} & {$[0]+[3]$} \\
\hline$[5]$ & & & & & & {$[1]+[4]$} \\
\hline
\end{tabular}


Table 9: Group $\mathbf{Z}_{3}^{2}$ covering the Fusion Table for $A_{2}$ of level $k=2$.

\begin{tabular}{|c|c|c|c|c|c|c|}
\hline$[\mathrm{a}] \times[\mathrm{b}]$ & $(0,0)$ & $(1,1)$ & $(2,2)$ & $\begin{array}{l}(1,2) \\
(2,1)\end{array}$ & $\begin{array}{l}(2,0) \\
(0,2)\end{array}$ & $\begin{array}{l}(1,0) \\
(0,1)\end{array}$ \\
\hline$(0,0)$ & $(0,0)$ & $(1,1)$ & $(2,2)$ & $\begin{array}{l}(1,2) \\
(2,1)\end{array}$ & $\begin{array}{l}(2,0) \\
(0,2)\end{array}$ & $\begin{array}{l}(1,0) \\
(0,1)\end{array}$ \\
\hline$(1,1)$ & & $(2,2)$ & $(0,0)$ & $\begin{array}{l}(2,0) \\
(0,2)\end{array}$ & $\begin{array}{l}(0,1) \\
(1,0)\end{array}$ & $\begin{array}{l}(2,1) \\
(1,2)\end{array}$ \\
\hline$(2,2)$ & & & $(1,1)$ & $\begin{array}{l}(0,1) \\
(1,0)\end{array}$ & $\begin{array}{l}(1,2) \\
(2,1)\end{array}$ & $\begin{array}{l}(0,2) \\
(2,0)\end{array}$ \\
\hline $\begin{array}{l}(1,2) \\
(2,1)\end{array}$ & & & & $\begin{array}{l}(2,1),(0,0) \\
0,0),(1,2)\end{array}$ & $\begin{array}{l}(0,2),(1,1) \\
(1,1),(2,0)\end{array}$ & $\begin{array}{l}(2,2),(0,1) \\
1,0),(2,2)\end{array}$ \\
\hline $\begin{array}{l}(2,0) \\
(0,2)\end{array}$ & & & & & $\begin{array}{l}(1,0),(2,2) \\
(2,2),(0,1)\end{array}$ & $\begin{array}{l}(0,0),(1,2) \\
(2,1),(0,0)\end{array}$ \\
\hline $\begin{array}{l}(1,0) \\
(0,1)\end{array}$ & & & & & & $\begin{array}{l}(2,0),(1,1) \\
1,1),(0,2)\end{array}$ \\
\hline
\end{tabular}

In the fusion table for $A_{2}$ of level $k=3$, part of which is shown below, we found a fusion coefficient greater than one, which our previous scheme could not handle. Our main problem has been to modify our method of covering fusion tables so as to account for such higher multiplicities. In doing so we found a surprising connection to a combinatorial problem which may be of some independent interest. In the next section we will set up the notation needed for this new point of view for $\hat{g}$ of type $A_{N-1}^{(1)}$, and see how the combinatorics enters the picture.

Table 10: Partial fusion Table for $A_{2}$ of level $k=3$.

\begin{tabular}{|c|c|c|c|c|}
\hline$[\mathrm{i}] \times[\mathrm{j}]$ & {$[0]$} & {$[1]$} & {$[2]$} & {$[9]$} \\
\hline$[0]$ & {$[0]$} & {$[1]$} & {$[2]$} & {$[9]$} \\
\hline$[1]$ & & {$[2]$} & {$[0]$} & {$[9]$} \\
\hline$[2]$ & & & {$[1]$} & {$[9]$} \\
\hline$[9]$ & & & & {$[0]+[1]+[2]+2[9]$} \\
\hline
\end{tabular}

\section{A New Approach Incorporating Higher Multiplicities}

Let $\hat{g}$ be the affine algebra of type $A_{N-1}^{(1)}$ built from $\mathbf{g}=s l_{N}$. Let $\lambda_{1}, \cdots, \lambda_{N-1}$ denote the fundamental weights of $\mathbf{g}$, and let

$$
P^{+}=\left\{\sum_{j=1}^{N-1} a_{j} \lambda_{j} \mid 0 \leq a_{j} \in \mathbf{Z}\right\}
$$

denote the dominant integral weights of $\mathbf{g}$. The irreducible modules $\hat{V}_{\Lambda}$ for $\hat{g}$ of level $k \geq 1$ are indexed by a dominant integral highest weight $\Lambda=k c+\lambda$ where

$$
\lambda=\sum_{j=1}^{N-1} a_{j} \lambda_{j} \in P^{+}
$$


satisfies the "level $k$ condition" $\sum_{j=1}^{N-1} a_{j} \leq k$. On level 1 , those dominant integral weights are the fundamental weights of $\hat{g}$,

$$
\Lambda_{0}=c, \Lambda_{1}=c+\lambda_{1}, \cdots, \Lambda_{N-1}=c+\lambda_{N-1}
$$

Then the irreducible modules on level $k$ are in one-to-one correspondence with the set of $N$-tuples of nonnegative integers $\left(i_{0}, i_{1}, \cdots, i_{N-1}\right)$ whose sum is $k$. Such an $N$-tuple corresponds to

$$
\begin{aligned}
\Lambda & =\sum_{j=0}^{N-1} i_{j} \Lambda_{j} \\
& =\left(\sum_{j=0}^{N-1} i_{j}\right) c+i_{1} \lambda_{1}+\cdots+i_{N-1} \lambda_{N-1} \\
& =k c+i_{1} \lambda_{1}+\cdots+i_{N-1} \lambda_{N-1} .
\end{aligned}
$$

Let $V_{\lambda}$ be the irreducible finite dimensional $g$-submodule of $\hat{V}_{\Lambda}$ generated by a highest weight vector. In the special case when $\Lambda=k \Lambda_{0}=k c$, that finite dimensional $\mathbf{g}$-module is $V_{0}$, the one dimensional trivial $\mathbf{g}$-module. Since $\mathbf{g}$ is semisimple, any finite dimensional g-module is completely reducible. Therefore, we can write the tensor product of irreducible g-modules

$$
V_{\lambda} \otimes V_{\mu}=\sum_{\nu \in P^{+}} M u l t_{\lambda, \mu}^{\nu} V_{\nu}
$$

as the direct sum of irreducible g-modules, including multiplicities. This decomposition is independent of the level $k$ and is part of the basic representation theory of $s l_{N}$. The fusion algebra for $\hat{g}$ on level $k$ has a basis in one-to-one correspondence with the irreducible $\hat{g}$-modules on level $k$, and its fusion rules are obtained by a rather subtle truncation of the above summation.

The fusion algebra for $A_{N-1}^{(1)}$ on level $k=1$ is rather trivial, with a basis of $N$ vectors labelled by the highest weights of the $N$ irreducible fundamental modules, and with fusion rules given by the group $\mathbf{Z}_{N}$, which is the weight lattice modulo the root lattice of $\mathbf{g}$. This means that $N_{\lambda, \mu}^{\nu}=\delta_{\lambda_{+} \mu, \nu}$ where the addition takes place in the quotient group of the weight lattice modulo the root lattice.

Let $G$ be the group $\mathbf{Z}_{N}^{k}$, and for each $N$-tuple of nonnegative integers

$$
\left(i_{0}, i_{1}, \cdots, i_{N-1}\right) \text { such that } i_{0}+i_{1}+\cdots+i_{N-1}=k
$$

define the subset of $G$

$$
P\left(i_{0}, i_{1}, \ldots, i_{N-1}\right)=\left\{x \in \mathbf{Z}_{N}^{k} \mid j \text { occurs exactly } i_{j} \text { times in } x, 0 \leq j \leq N-1\right\}
$$

Then the cardinality of the subset $P\left(i_{0}, i_{1}, \ldots, i_{N-1}\right)$ is

$$
\left(\begin{array}{c}
k \\
i_{0}, i_{1}, \ldots, i_{N-1}
\end{array}\right)
$$


and $G$ is the disjoint union of these subsets. For example, with $N=3$ and $k=2$, a bijection between these subsets and a basis of the $s l_{3}$ fusion algebra on level 2 is:

$$
\begin{aligned}
& {[0]=\{(0,0)\}=P(2,0,0)} \\
& {[1]=\{(1,1)\}=P(0,2,0)} \\
& {[2]=\{(2,2)\}=P(0,0,2)} \\
& {[3]=\{(1,2),(2,1)\}=P(0,1,1)} \\
& {[4]=\{(2,0),(0,2)\}=P(1,0,1)} \\
& {[5]=\{(1,0),(0,1)\}=P(1,1,0)}
\end{aligned}
$$

We can now start to describe our new method of covering the fusion algebra $F=F(g, k)$ for $\mathbf{g}=s l_{N}$ on level $k$ by the group $G=\mathbf{Z}_{N}^{k}$. First note that the symmetric group $S_{k}$ acts on $G$ by permuting the $k$-tuples. For $a \in G$, let $\mathcal{O}_{a}=[a]$ denote the orbit of $a$ under this action and let $\mathcal{O}$ be the set of all such orbits. It is easy to see that these orbits are precisely the subsets $P\left(i_{0}, i_{1}, \ldots, i_{N-1}\right)$ defined above, and that each orbit contains a unique representative in the "standard" form

$$
\left(0^{i_{0}}, 1^{i_{1}}, \cdots,(N-1)^{i_{N-1}}\right)
$$

where the exponent indicates the number of repetitions of the base. For $[a],[b],[c] \in$ $\mathcal{O}$ we wish to define a fusion coefficient $N_{[a],[b]}^{[c]}$ in terms of the group $G$ so that the resulting product

$$
[a] \cdot[b]=\sum_{[c] \in \mathcal{O}} N_{[a],[b]}^{[c]}[c]
$$

defines a fusion algebra isomorphic to the fusion algebra $F$. It is clear that the distinguished element $\Omega$ of the fusion algebra should correspond to [0] and that the conjugate of $[c]$ should be $[-c]$. Then we can understand the fusion coefficients by studying the completely symmetric coefficients

$$
N_{[a],[b],[c]}=N_{[a],[b]}^{[-c]} .
$$

We may assume that

$$
\begin{aligned}
a & =\left(0^{\alpha_{0}}, 1^{\alpha_{1}}, \cdots,(N-1)^{\alpha_{N-1}}\right) \\
b & =\left(0^{\beta_{0}}, 1^{\beta_{1}}, \cdots,(N-1)^{\beta_{N-1}}\right) \\
c & =\left(0^{\gamma_{0}}, 1^{\gamma_{1}}, \cdots,(N-1)^{\gamma_{N-1}}\right)
\end{aligned}
$$

are in standard form. The exponents are nonnegative integers satisfying

$$
\sum_{i=0}^{N-1} \alpha_{i}=\sum_{i=0}^{N-1} \beta_{i}=\sum_{i=0}^{N-1} \gamma_{i}=k .
$$

The group $S_{k}$ acts on

$$
T([a],[b],[c])=\{(x, y, z) \in[a] \times[b] \times[c] \mid x+y+z=0\}
$$

which decomposes into a finite number of orbits under that action. We wish to determine that number of orbits, and show how it is related to the fusion coefficient $N_{[a],[b],[c]}$. We will show how to do this for $N=2$ and for $N=3$ in the next two sections, and we hope that future work will give the relationship for all ranks. 
Definition. Let $M([a],[b],[c])$ be the number of orbits of $T([a],[b],[c])$ under the action of $S_{k}$.

Each orbit of $T([a],[b],[c])$ under $S_{k}$ has representatives whose third component is in the standard form, and in fact, we may assume that $z=c$ is in that standard form. This makes it clear that the above counting problem is equivalent to counting the number of orbits of

$$
T_{c}([a],[b])=\{(x, y) \in[a] \times[b] \mid x+y+c=0\}
$$

under the action of the stabilizer of $c$ in $S_{k}, \operatorname{Stab}_{S_{k}}(c)=\left\{\sigma \in S_{k} \mid \sigma(c)=c\right\}$. The structure of $S t a b_{S_{k}}(c)$ is obviously the product of symmetric groups $S_{\gamma_{0}} \times S_{\gamma_{1}} \times$ $\cdots \times S_{\gamma_{N-1}}$ since each subset of $\gamma_{j}$ occurences of $j$ can be freely permuted without changing $c$. We now write

$$
x=\left(x_{0}, x_{1}, \ldots, x_{N-1}\right) \quad \text { and } \quad y=\left(y_{0}, y_{1}, \ldots, y_{N-1}\right)
$$

where $x_{i}$ is the part of $x$ in the positions where $c$ has $i$ 's and where $y_{i}$ is the part of $y$ in the positions where $c$ has $i$ 's. By the action of the stabilizer of $c$, we can find a unique representative of the orbit of that stabilizer so that each

$$
x_{i}=\left(0^{k_{i 0}}, 1^{k_{i 1}}, \ldots,(N-1)^{k_{i, N-1}}\right)
$$

is in standard form. This defines the nonnegative integers $k_{i j}$ for $0 \leq i, j \leq N-1$, which we use to form the matrix $K=\left[k_{i j}\right]$. Then by the sum property, $x_{i}+y_{i}+c_{i}=$ $\left(0^{\gamma_{i}}\right)$, we must have

$$
y_{i}=\left((N-i)^{k_{i 0}},(N-1-i)^{k_{i 1}}, \ldots,(1-i)^{k_{i, N-1}}\right) .
$$

The $x$ and $y$ that have these forms are a system of distinct representatives of the orbits, thus the number of such pairs $(x, y)$ is the number $M([a],[b],[c])$. Below is a picture of the three vectors $x_{i}, y_{i}$ and $c_{i}$.

$$
\begin{aligned}
& x_{i}=\left(\begin{array}{clccc}
0^{k_{i 0}} & , & 1^{k_{i 1}} & , \ldots, & (N-1)^{k_{i, N-1}}
\end{array}\right) \\
& y_{i}=\left(\begin{array}{ccccc}
(N-i)^{k_{i 0}} & , & (N-1-i)^{k_{i 1}} & , \ldots & (1-i)^{k_{i, N-1}}
\end{array}\right) \\
& c_{i}=\left(\begin{array}{ccccc}
i^{k_{i 0}} & , & i^{k_{i 1}} & , \ldots, & i^{k_{i, N-1}}
\end{array}\right)
\end{aligned}
$$

The $3 N$ equations which must be satisfied by the $N^{2}$ unknowns, $0 \leq k_{i j} \in \mathbf{Z}$, are

$$
\begin{aligned}
& \sum_{j=0}^{N-1} k_{i j}=\gamma_{i}, \quad \text { for } 0 \leq i \leq N-1 \\
& \sum_{i=0}^{N-1} k_{i j}=\alpha_{j}, \quad \text { for } 0 \leq j \leq N-1 \\
& \sum_{i=0}^{N-1} k_{i, l-i}=\beta_{N-l}, \quad \text { for } 1 \leq l \leq N .
\end{aligned}
$$

The first two equations are easy to understand from the definitions above as specifications of the sums in each row and column. But the third one, which specifies 
the sum of each upright diagonal (wrapped as if the matrix were toroidal), may need a little more consideration. When we write out the entries

$$
\begin{aligned}
& y_{0}=\begin{array}{llll}
(N)^{k_{00}} & (N-1)^{k_{01}} & \ldots & (1)^{k_{0, N-1}}
\end{array} \\
& y_{1}=\left(\begin{array}{llll}
(N-1)^{k_{10}} & (N-2)^{k_{11}} & \ldots & (0)^{k_{1, N-1}}
\end{array}\right. \\
& y_{2}=(N-2)^{k_{20}}(N-3)^{k_{21}} \ldots \quad(N-1)^{k_{2, N-1}} \\
& \vdots \\
& y_{N-1}=(1)^{k_{N-1,0}} \quad(0)^{k_{N-1,1}} \quad \ldots \quad(2)^{k_{N-1, N-1}}
\end{aligned}
$$

we see that the base of the exponent $k_{i j}$ is $N-(i+j)$ modulo $N$, so the total number of occurences of base $N-l$ in $y$ is that upright diagonal sum.

There are $N^{2}$ variables, and there are $3 N$ equations, but the consistency of sums of different types means that 2 equations (of different types, say one column and one diagonal equation) are redundant. Therefore the dimension of the solution space (over Q) is $N^{2}-(3 N-2)=(N-1)(N-2)$, which is 0 for $N=2,2$ for $N=3$, and 6 for $N=4$.

\section{The case of $N=2$.}

For $N=2$ with

$$
a=\left(0^{\alpha_{0}}, 1^{\alpha_{1}}\right) \quad b=\left(0^{\beta_{0}}, 1^{\beta_{1}}\right) \quad c=\left(0^{\gamma_{0}}, 1^{\gamma_{1}}\right)
$$

and

$$
\alpha_{0}+\alpha_{1}=\beta_{0}+\beta_{1}=\gamma_{0}+\gamma_{1}=k
$$

we have the equations

$$
\begin{array}{ll}
k_{00}+k_{01}=\gamma_{0} & k_{10}+k_{11}=\gamma_{1} \\
k_{00}+k_{10}=\alpha_{0} & k_{01}+k_{11}=\alpha_{1} . \\
k_{01}+k_{10}=\beta_{1} & k_{00}+k_{11}=\beta_{0}
\end{array}
$$

Three of these involving $k_{00}$ allow us to write

$$
K=\left[\begin{array}{ll}
k_{00} & k_{01} \\
k_{10} & k_{11}
\end{array}\right]=\left[\begin{array}{cc}
k_{00} & \gamma_{0}-k_{00} \\
\alpha_{0}-k_{00} & \beta_{0}-k_{00}
\end{array}\right],
$$

so if a solution exists, then $k_{00}$ determines the matrix uniquely. Each of the other equations is equivalent to

$$
2 k_{00}=\alpha_{0}+\beta_{0}+\gamma_{0}-k=2 k-\left(\alpha_{1}+\beta_{1}+\gamma_{1}\right)
$$

so

$$
0 \leq k_{00}=k-\frac{1}{2}\left(\alpha_{1}+\beta_{1}+\gamma_{1}\right) \in \mathbf{Z}
$$

imposes the requirement that

$$
\frac{1}{2}\left(\alpha_{1}+\beta_{1}+\gamma_{1}\right) \in \mathbf{Z} .
$$

Since all entries of the matrix are nonnegative, we have $0 \leq k_{00} \leq \min \left(\alpha_{0}, \beta_{0}, \gamma_{0}\right)$ so

$$
0 \leq \alpha_{0}+\beta_{0}+\gamma_{0}-k \leq 2 \min \left(\alpha_{0}, \beta_{0}, \gamma_{0}\right)
$$

which is equivalent to

$$
\max \left(2 \alpha_{1}, 2 \beta_{1}, 2 \gamma_{1}\right) \leq \alpha_{1}+\beta_{1}+\gamma_{1} \leq 2 k .
$$


Rewriting this as

$$
\max \left(\alpha_{1}-\beta_{1}, \beta_{1}-\alpha_{1}, 2 \gamma_{1}-\left(\alpha_{1}+\beta_{1}\right)\right) \leq \gamma_{1} \leq 2 k-\left(\alpha_{1}+\beta_{1}\right)
$$

and using $2 \gamma_{1}-\left(\alpha_{1}+\beta_{1}\right) \leq \gamma_{1}$ iff $\gamma_{1} \leq \alpha_{1}+\beta_{1}$, we get the condition

$$
\max \left(\alpha_{1}-\beta_{1}, \beta_{1}-\alpha_{1}\right) \leq \gamma_{1} \leq \min \left(2 k-\left(\alpha_{1}+\beta_{1}\right), \alpha_{1}+\beta_{1}\right)
$$

which is equivalent to

$$
\left|\alpha_{1}-\beta_{1}\right| \leq \gamma_{1} \leq \alpha_{1}+\beta_{1} \quad \text { and } \quad \alpha_{1}+\beta_{1}+\gamma_{1} \leq 2 k .
$$

if

TheOREm 3. With notation as above, for $1 \leq k \in \mathbf{Z}$, we have $M([a],[b],[c])=1$

$$
\left|\alpha_{1}-\beta_{1}\right| \leq \gamma_{1} \leq \alpha_{1}+\beta_{1}, \quad \alpha_{1}+\beta_{1}+\gamma_{1} \leq 2 k \quad \text { and } \quad \frac{1}{2}\left(\alpha_{1}+\beta_{1}+\gamma_{1}\right) \in \mathbf{Z},
$$

$M([a],[b],[c])=0$ otherwise.

Corollary. When $N=2$, with notation as above, and with $a_{1}=\alpha_{1} / 2$, $b_{1}=\beta_{1} / 2$ and $c_{1}=\gamma_{1} / 2$, we have $M([a],[b],[c])=N_{\left[a_{1}\right],\left[b_{1}\right],\left[c_{1}\right]}$.

ProOF. The element $a=\left(0^{\alpha_{0}}, 1^{\alpha_{1}}\right) \in \mathbf{Z}_{2}^{k}$ corresponds to a level $k$ highest weight $\Lambda=\alpha_{0} \Lambda_{0}+\alpha_{1} \Lambda_{1}=k c+\alpha_{1} \lambda_{1}$, for $0 \leq \alpha_{1} \leq k$ integral. The associated irreducible highest weight $s l_{2}$-module is then $V_{\alpha_{1} / 2}$ if we use the spin $a_{1}=\alpha_{1} / 2$ to label the module. The labels for the $s l_{2}$-modules associated with $b$ and $c$ are $b_{1}=\beta_{1} / 2$ and $c_{1}=\gamma_{1} / 2$. Since $c=-c \in \mathbf{Z}_{2}^{k}, N_{\left[a_{1}\right],\left[b_{1}\right],\left[c_{1}\right]}=N_{\left[a_{1}\right],\left[b_{1}\right]}^{\left[c_{1}\right]}$, and the conditions in the theorem above translate exactly into the conditions of the fusion rules for $s l_{2}$.

\section{The case of $N=3$.}

For $N=3$, after using two column sums, two row sums and one diagonal sum to rewrite the entries in the last row and column of $K$, we have

$$
K=\left[\begin{array}{ccc}
k_{00} & k_{01} & \gamma_{0}-k_{00}-k_{01} \\
k_{10} & k_{11} & \gamma_{1}-k_{10}-k_{11} \\
\alpha_{0}-k_{00}-k_{10} & \alpha_{1}-k_{01}-k_{11} & \beta_{2}-k_{01}-k_{10}
\end{array}\right] .
$$

This leaves 4 more equations:

$$
\begin{aligned}
{\left[\alpha_{0}-k_{00}-k_{10}\right]+\left[\alpha_{1}-k_{01}-k_{11}\right]+\left[\beta_{2}-k_{01}-k_{10}\right] } & =\gamma_{2}, \\
{\left[\gamma_{0}-k_{00}-k_{01}\right]+\left[\gamma_{1}-k_{10}-k_{11}\right]+\left[\beta_{2}-k_{01}-k_{10}\right] } & =\alpha_{2}, \\
k_{00}+\left[\gamma_{1}-k_{10}-k_{11}\right]+\left[\alpha_{1}-k_{01}-k_{11}\right] & =\beta_{0}, \\
{\left[\gamma_{0}-k_{00}-k_{01}\right]+k_{11}+\left[\alpha_{0}-k_{00}-k_{10}\right] } & =\beta_{1} .
\end{aligned}
$$

Using the relation

$$
k=\alpha_{0}+\alpha_{1}+\alpha_{2}=\beta_{0}+\beta_{1}+\beta_{2}=\gamma_{0}+\gamma_{1}+\gamma_{2}
$$

we find that two of the four equations are redundant, and the system is equivalent to

$$
\begin{aligned}
k_{00}+2 k_{01}+2 k_{10}+k_{11} & =k-\alpha_{2}+\beta_{2}-\gamma_{2}, \\
3\left(k_{00}-k_{11}\right) & =\left(\alpha_{0}+\beta_{0}+\gamma_{0}\right)-\left(\alpha_{1}+\beta_{1}+\gamma_{1}\right) .
\end{aligned}
$$


Letting

$$
A=\frac{1}{3}\left[\left(\alpha_{0}+\beta_{0}+\gamma_{0}\right)-\left(\alpha_{1}+\beta_{1}+\gamma_{1}\right)\right]
$$

the solutions to the linear system above are

$$
k_{00}=k_{11}+A \quad k_{01}=-k_{10}-k_{11}+A+\alpha_{1}-\beta_{0}+\gamma_{1}
$$

where $k_{10}$ and $k_{11}$ are the "free variables". In fact, because the entries of the matrix $K$ are nonnegative integers, we have $A \in \mathbf{Z}$ and the following nine inequalities:

$$
\begin{gathered}
0 \leq k_{00} \quad 0 \leq k_{01} \quad 0 \leq k_{10} \quad 0 \leq k_{11} \quad k_{01}+k_{10} \leq \beta_{2} \\
k_{00}+k_{10} \leq \alpha_{0} \quad k_{01}+k_{11} \leq \alpha_{1} \quad k_{00}+k_{01} \leq \gamma_{0} \quad k_{10}+k_{11} \leq \gamma_{1} .
\end{gathered}
$$

These translate into the following inequalities on the free variables $k_{10}$ and $k_{11}$ :

$$
\begin{gathered}
-A \leq k_{11} \quad k_{10}+k_{11} \leq A+\alpha_{1}-\beta_{0}+\gamma_{1} \quad 0 \leq k_{10} \quad 0 \leq k_{11} \\
k_{10}+k_{11} \leq \alpha_{0}-A \quad A-\beta_{0}+\gamma_{1} \leq k_{10} \quad 2 A+\alpha_{1}-\beta_{0}+\gamma_{1}-\gamma_{0} \leq k_{10} \\
k_{10}+k_{11} \leq \gamma_{1} \quad A+\alpha_{1}-\beta_{0}-\beta_{2}+\gamma_{1} \leq k_{11} .
\end{gathered}
$$

Combining these, we can say that

$$
\begin{gathered}
C=\max \left(0, A-\beta_{0}+\gamma_{1}, 2 A+\alpha_{1}-\beta_{0}+\gamma_{1}-\gamma_{0}\right) \leq k_{10} \\
D=\max \left(-A, 0, A+\alpha_{1}-\beta_{0}-\beta_{2}+\gamma_{1}\right) \leq k_{11} \\
k_{10}+k_{11} \leq \min \left(A+\alpha_{1}-\beta_{0}+\gamma_{1}, \alpha_{0}-A, \gamma_{1}\right)=E .
\end{gathered}
$$

It is clear that when $E-C \geq D$ and $A \in \mathbf{Z}$, these constraints define a "45-4590 " triangle in the first quadrant of the $k_{10} k_{11}$-plane whose vertices have integral coordinates. The number $M([a],[b],[c])$ is exactly the number of integral points within that triangle, including its boundary. The number of points on one leg of the triangle is clearly $E-D-C+1$ so that $M([a],[b],[c])$ is a triangular number.

THEOREM 4. With notation as above, for $1 \leq k \in \mathbf{Z}$, we have

$$
M([a],[b],[c])=\frac{(E-D-C+1)(E-D-C+2)}{2}=\left(\begin{array}{c}
E-D-C+2 \\
2
\end{array}\right) .
$$

We will now show that $E-D-C+1$ is equal to the fusion rule coefficient $N_{[a],[b],[c]}$ by using the theorem of [BMW]. The level $k$ highest weights, $\lambda, \mu$ and $\nu$, referred to in the $[\mathbf{B M W}]$ theorem, correspond to $a, b$ and $c$ in $\mathbf{Z}_{3}^{k}$ so that

$$
\lambda=\alpha_{1} \lambda_{1}+\alpha_{2} \lambda_{2}, \quad \mu=\beta_{1} \lambda_{1}+\beta_{2} \lambda_{2}, \quad \nu=\gamma_{1} \lambda_{1}+\gamma_{2} \lambda_{2} .
$$

We have

so that

$$
\begin{aligned}
& \mathcal{A}=\frac{1}{3}\left[2\left(\alpha_{1}+\beta_{1}+\gamma_{1}\right)+\left(\alpha_{2}+\beta_{2}+\gamma_{2}\right)\right], \\
& \mathcal{B}=\frac{1}{3}\left[\left(\alpha_{1}+\beta_{1}+\gamma_{1}\right)+2\left(\alpha_{2}+\beta_{2}+\gamma_{2}\right)\right],
\end{aligned}
$$

$$
\mathcal{A}=k-A, \quad 2 \mathcal{A}-\mathcal{B}=\alpha_{1}+\beta_{1}+\gamma_{1}, \quad 2 \mathcal{B}-\mathcal{A}=\alpha_{2}+\beta_{2}+\gamma_{2},
$$

and

$$
\mathcal{A}+\mathcal{B}=\left(\alpha_{1}+\beta_{1}+\gamma_{1}\right)+\left(\alpha_{2}+\beta_{2}+\gamma_{2}\right)
$$


The variables used in the $[\mathbf{B M W}]$ theorem are

$$
\begin{aligned}
k_{0}^{\max } & =\min (\mathcal{A}, \mathcal{B}) \\
k_{0}^{\min } & =\max \left(\alpha_{1}+\alpha_{2}, \beta_{1}+\beta_{2}, \gamma_{1}+\gamma_{2}, \mathcal{A}-\min \left(\alpha_{1}, \beta_{1}, \gamma_{1}\right), \mathcal{B}-\min \left(\alpha_{2}, \beta_{2}, \gamma_{2}\right)\right) \\
& =\max \left(k-\min \left(\alpha_{0}, \beta_{0}, \gamma_{0}\right), \mathcal{A}-\min \left(\alpha_{1}, \beta_{1}, \gamma_{1}\right), \mathcal{B}-\min \left(\alpha_{2}, \beta_{2}, \gamma_{2}\right)\right) .
\end{aligned}
$$

The $[\mathbf{B M W}]$ theorem says that the fusion coefficient

$$
N_{\lambda, \mu, \nu}^{(k)}=\min \left(k_{0}^{\max }, k\right)-k_{0}^{\min }+1
$$

if the conditions

$$
k_{0}^{\min } \leq k_{0}^{\max }, \quad k_{0}^{\min } \leq k \quad \text { and } \quad \mathcal{A}, \mathcal{B} \in \mathbf{Z}
$$

are satisfied, and the coefficient is zero otherwise. Using the formulas above, it is straightforward to check that

$$
\begin{gathered}
C=\max \left(0, \beta_{1}+\beta_{2}+\gamma_{1}-\mathcal{A}, \mathcal{B}-\mathcal{A}-\alpha_{2}+\gamma_{1}\right), \\
D=\mathcal{A}-\min (\mathcal{A}, \mathcal{B}, k)=\mathcal{A}-\min \left(k_{0}^{\max }, k\right), \\
E=\mathcal{A}-\max \left(\alpha_{1}+\alpha_{2}, \mathcal{A}-\gamma_{1}, \mathcal{B}-\beta_{2}\right) .
\end{gathered}
$$

Two identities useful for checking this are

$$
\begin{aligned}
& 2 A+\alpha_{1}-\beta_{0}+\gamma_{1}-\gamma_{0}=\mathcal{B}-\mathcal{A}-\alpha_{2}+\gamma_{1}, \\
& A+\alpha_{1}-\beta_{0}-\beta_{2}+\gamma_{1}=\mathcal{B}-\left(\alpha_{2}+\beta_{2}+\gamma_{2}\right) .
\end{aligned}
$$

From these expressions for $C, D$ and $E$ we can now compute

$$
\begin{gathered}
E-D-C+1=\min \left(k_{0}^{\max }, k\right)+1- \\
\left\{\max \left(0, \beta_{1}+\beta_{2}-\mathcal{A}+\gamma_{1}, \mathcal{B}-\mathcal{A}-\alpha_{2}+\gamma_{1}\right)+\max \left(\alpha_{1}+\alpha_{2}, \mathcal{A}-\gamma_{1}, \mathcal{B}-\beta_{2}\right)\right\} \\
=\min \left(k_{0}^{\max }, k\right)+1- \\
\max \left(\alpha_{1}+\alpha_{2}, \mathcal{A}-\gamma_{1}, \mathcal{B}-\beta_{2}, \mathcal{B}-\gamma_{2}, \beta_{1}+\beta_{2}, \mathcal{A}-\alpha_{1}, \mathcal{A}-\beta_{1}, \mathcal{B}-\alpha_{2}, \gamma_{1}+\gamma_{2}\right) \\
=\min \left(k_{0}^{\max }, k\right)+1-k_{0}^{\min } .
\end{gathered}
$$

We also have

$$
E-C=\mathcal{A}-k_{0}^{\min }
$$

so the condition $E-C \geq D$ is equivalent to the condition

$$
\min \left(k_{0}^{\max }, k\right) \geq k_{0}^{\min },
$$

that is,

$$
k_{0}^{\max } \geq k_{0}^{\min } \text { and } k \geq k_{0}^{\min } .
$$

It is clear that $A \in \mathbf{Z}$ iff $\mathcal{A} \in \mathbf{Z}$ iff $\mathcal{B} \in \mathbf{Z}$ since $\mathcal{A}=k-A$ and $\mathcal{A}+\mathcal{B} \in \mathbf{Z}$. We have shown that the conditions for the fusion coefficient to be nonzero are equivalent to the conditions for $M([a],[b],[c]) \neq 0$. We have now proved our main result. 
TheOREM 5. For $N=3$, the number of orbits of $T([a],[b],[c])$ under the action of $S_{k}$ is related to the fusion coefficient $N_{[a],[b],[c]}$ by

$$
M([a],[b],[c])=\left(\begin{array}{c}
N_{[a],[b],[c]}+1 \\
2
\end{array}\right) .
$$

Another form of the conditions and number of orbits can be given as follows. For $i, j, l, r \in\{0,1,2\}$ taken modulo 3 , let

$$
s_{i j l}=\alpha_{i}+\beta_{j}+\gamma_{l},
$$

and

$$
D_{i j}^{r}=s_{i, i+r, i+2 r}-s_{j, j+r, j+2 r} .
$$

Then, using the fact that for $(i, j, l)$ any permutation of $(0,1,2)$,

$$
s_{i, i+r, i+2 r}+s_{l, l+r, l+2 r}=3 k-s_{j, j+r, j+2 r}
$$

we get that

$$
D_{i j}^{r}-D_{j l}^{r}=3\left(k-s_{j, j+r, j+2 r}\right)
$$

so

$$
D_{i j}^{r} \equiv D_{j l}^{r} \quad(\bmod 3)
$$

In fact, we have that $D_{01}^{0} \equiv D_{12}^{0} \equiv D_{20}^{0} \equiv D_{01}^{1} \equiv D_{12}^{1} \equiv D_{20}^{1} \equiv D_{01}^{2} \equiv D_{12}^{2} \equiv D_{20}^{2}$ $(\bmod 3)$. This makes the necessary condition written below $(3 \mid D)$ for existence of a solution, independent of which $D_{i j}^{r}$ one checks for divisibility by 3 .

Theorem 6. [Zaslavsky] Let $D$ be any $D_{i, i+1}^{r}$ defined above and define $m$ by

$$
3 m=\min \left(s_{000}, s_{111}, s_{222}\right)+\min \left(s_{012}, s_{120}, s_{201}\right)+\min \left(s_{021}, s_{102}, s_{210}\right)-2 k .
$$

If $3 \mid D$ and $m \geq 0$ then we have $m \in \mathbf{Z}$ and $M([a],[b],[c])=\left(\begin{array}{c}m+2 \\ 2\end{array}\right)$. Otherwise, $M([a],[b],[c])=0$.

\section{References}

[AFW] F. Akman, A. Feingold, M. Weiner, Minimal model fusion rules from 2-groups, Letters in Mathematical Physics 40 (1997), 159-169.

[BMW] L. Bégin, P. Mathieu, M. A. Walton, $\widehat{s u}(3)_{k}$ fusion coefficients, Modern Physics Letters A7 (1992), 3255-3266.

[BKMW] L. Bégin, A. N. Kirillov, P. Mathieu, M. A. Walton, Berenstein-Zelevinsky triangles, elementary couplings and fusion rules, Lett. Math. Phys. 28 (1993), 257-268.

[DL] C. Dong, J. Lepowsky, Abelian intertwining algebras - a generalization of vertex operator algebras, Proc. Symp. Pure Math, 56, Part 2, Algebraic Groups and Their Generalizations (William J. Haboush and Brian J. Parshall, ed.), Amer. Math. Soc., Providence, RI, 1994, pp. 261-293.

[Fe] A. J. Feingold, Constructions of vertex operator algebras, Proc. Symp. Pure Math., 56, Part 2, Algebraic Groups and Their Generalizations (William J. Haboush and Brian J. Parshall, ed.), Amer. Math. Soc., Providence, RI, 1994, pp. 317-336.

[FFR] A. J. Feingold, I. B. Frenkel, J. F. X. Ries, Spinor Construction of Vertex Operator Algebras, Triality and $E_{8}^{(1)}$, Contemp. Math., 121, Amer. Math. Soc., Providence, RI, 1991.

[FRW] A. Feingold, J. F. X. Ries, M. D. Weiner, Spinor construction of the $c=\frac{1}{2}$ minimal model, Moonshine, The Monster, and Related Topics, Contemporary Math., Vol 193 (Chongying Dong and Geoffrey Mason, eds.), Amer. Math. Soc., Providence, RI, 1995, pp. $45-92$. 
[FHL] I. B. Frenkel, Yi-Zhi Huang, J. Lepowsky, On axiomatic approaches to vertex operator algebras and modules, Memoirs Amer. Math. Soc., 104, No. 594, Amer. Math. Soc., Providence, RI, 1993.

[FLM] I. B. Frenkel, J. Lepowsky, A. Meurman, Vertex Operator Algebras and the Monster, Pure and Applied Math., 134, Academic Press, Boston, 1988.

[FZ] I. B. Frenkel, Y. Zhu, Vertex operator algebras associated to representations of affine and Virasoro algebras, Duke Math. J. 66 (1992), 123-168.

[Fu] J. Fuchs, Fusion rules in conformal field theory, Fortsch. Phys. 42 (1994), 1-48.

[GO] P. Goddard, D. I. Olive, Kac-Moody and Virasoro algebras in relation to quantum physics, Internat. J. Mod. Phys. A, Vol. 1, No. 2 (1986), 303-414.

[KR] V. G. Kac, A. K. Raina, Bombay Lectures on Highest Weight Representations, World Scientific, Singapore, 1987.

[TK] A. Tsuchiya, Y. Kanie, Vertex operators in conformal field theory on $\mathbb{P}^{1}$ and monodromy representations of braid group, Conformal Field Theory and Solvable Lattice Models, Adv. Studies in Pure Math., vol. 16, Academic Press, New York, 1988, pp. 297-372.

[T] G. Tudose, A special case of sl(n)-fusion coefficients, ArXiv:math.CO/0008034 (2000).

[Wa] Weiqiang Wang, Rationality of Virasoro vertex operator algebras, Duke Math. J. IMRN, Vol. 71, No. 1 (1993), 197-211.

[We] M. D. Weiner, Bosonic construction of vertex operator para-algebras from symplectic affine Kac-Moody algebras, Memoirs Amer. Math. Soc. 135 (1998).

Dept. of Math. Sci., The State University of New York, Binghamton, New York 13902-6000

E-mail address: alex@math.binghamton.edu

Math. Dept., Pennsylvania State University, Altoona, Pennsylvania 16601

E-mail address: mdw8@psu.edu 